NBSIR 75-917

\title{
A Multiple-Chamber Humidity Apparatus
}

Lewis Greenspan

Institute for Basic Standards National Bureau of Standards

Washington, D. C. 20234

December 1975

Final Report

Prepared for

Department of the Army

U.S. Army Natick Development Center

Natick, Massachusetts 01760 



\section{A MULTIPLE-CHAMBER HUMIIDITY APPARATUS}

Lewis Greenspan

Institute for Basic Standards

National Bureau of Standards

Washington, D. C. 20234

December 1975

Final Report

Prepared for

Department of the Army

U. S. Army Natick Development Center

Natick, Massachusetts 01760

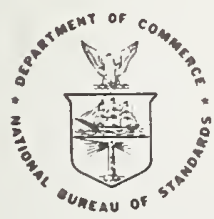

U.S. DEPARTMENT OF COMMERCE, Rogers C.B. Morton, Secretary

James A. Baker, III, Under Secretary

Dr. Betsy Ancker-Johnson, Assistant Secretary for Science and Technology

NATIONAL BUREAU OF STANDARDS, Emest Ambler, Acting Director 
A Multiple Chamber Humidity Apparatus

Abstract

An apparatus has been developed for studying the growth of microblological organisms on food under controlled conditions of humidity and temperature. This apparatus contains twenty-eight individual humidity chambers within a temperature controlled bath. A wide range of humidities can be provided within the individual chambers by means of saturated salt solutions. These chambers can be maintained at stable temperatires from 5 to $50{ }^{\circ} \mathrm{C}$ with a constancy and uniformity within $\pm .02 \mathrm{deg} C$. Individual chambers may be convenient $1 \mathrm{y}$ removed or changed without affecting the other chambers. 


\section{Introduction}

This report describes equipment intended for the study of microbiological growth as a function of temperature and relative humidity. In order that these studies may be performed in a reasonable length of time, a multiplicity of chambers of different humidities is required. A practical method of providing a number of different humidities simultaneously is by means of saturated salt solutions.

Any water solution has an equilibrium water vapor pressure which is a function of the type of solute, the solute concentration and the temperature of the solution. When a closed 1sothermal environment, containing no sources or sinks for water vapor, is exposed to such a solution, a fixed humidity will be established within the environment which is in equilibrium with the solution.

The equilibrium water vapor pressure is known for many solutions as a function of temperature and concentration. Examples of such solutions are aqueous sulfuric acld solutions and glycerine and water solutions. For these, a knowledge of the concentration and temperature provides a knowledge of the surrounding humidity. The problem with such solutions is that the concentration must be measured and even the process of equilibrating a chamber can change this concentration unless the quantity of solution is large with respect to the chamber volume. Obviously any humidity sources or sinks will alter the concentration. 
Water solutions of various salts also provide equilibrium water vapor pressures as a function of concentration and temperature. Many of these salts have a finite solubility at a fixed temperature and therefore a saturated solution of these salts is a solution of fixed concentration at a fixed temperature.

If the saturated salt solution contains excess salt, the concentration will remain constant at any fixed temperature, even in the presence of sources or sinks for water vapor as long as there is any liquid in the solution and as long as any undissolved solute remains. For these saturated salt solutions, the equilibrium water vapor pressure can be known by merely knowing the temperature and the type of solute - no measure of concentration being required. Such saturated salt solutions appear very appropriate for use in this apparatus, due to their ease of use and their ability to maintain a constant humidity despite the sorption by or desorption from the test samples. 
The Multiple-Chamber Humidity Apparatus consists of a constant temperature bath, individual humidity chambers and a rack to hold the chambers within the bath. Also included are the auxiliary equipment necessary to operate the constant temperature bath at temperatures from 5 to $50{ }^{\circ} \mathrm{C}$. All equipment is mounted on a three-shelf open cart $122 \mathrm{~cm}$ long and $61 \mathrm{~cm}$ wide. The maximum height occupied by the equipment on the cart is $170 \mathrm{~cm}$.

The bath consists of a stainless steel rectangular parallelepiped with internal dimensions of 89 by $48 \mathrm{~cm}$ and a depth of $29 \mathrm{~cm}$. This provides an internal volume of approximately 0.12 cubic meter. Figure 1 shows an external view of the bath on its cart.

At the bottom of the bath are two U-shaped heating units extending the length of the bath. Also at the bottom of the bath at one end is a magnetic agitator consisting of a weighted vane which is alternately drawn into an unstable position by means of an electromagnet below the bottom surface of the bath and released. The frequency and amplitude of the vane oscillation is controllable. The effect of the agitation is to cause a wave action in the bath fluid, providing additional circulation over the cooling coils. 
The humidity chambers are wide mouth glass containers of approximately $450 \mathrm{~cm}^{3}$ capacity with a coarse external screw thread at the open end. Each container is sealed by means of a stainless steel lid, $6 \mathrm{~cm}$ in diameter which contains a viton gasket, $0.4 \mathrm{~cm}$ wide by $0.08 \mathrm{~cm}$ thick inset on the outer edge. The viton gasket seals against the glass lip of the container. The lid is held against the glass container by means of a cap which is screwed against the lid by engaging the threads of the glass container with internal threads in the cap. Suspended from the lid by means of four stainless steel chains are two stainless steel shelves for holding test material. Figure 2 is a view of two chambers, one sealed and the other open.

The chamber rack consists of two identical horizontal stainless steel plates of $.6 \mathrm{~cm}$ thickness which $\mathrm{f} i 11$ the constant temperature bath. One plate sits on four $53 / 4 \mathrm{~cm}$ long stainless steel legs and the other is joined to the first plate by four stainless steel studs of $91 / 2 \mathrm{~cm}$ length. The legs of the rack sit on the bottom of the bath with the top surface of the rack being at a depth of $8 \mathrm{~cm}$ into the bath. Figure 3 is a view of the apparatus showing the rack in place in the bath. 
Both plates of the rack have 28 circular holes of $8.1 \mathrm{~cm}$ diameter on $10 \mathrm{~cm}$ centers, which act as support for a like number of humidity chambers. At one corner of the plates is a $12 \mathrm{~cm}$ square hole which provides the space for a centrifugal type stirrer and a resistance thermometer. A stainless steel spring stretches across the center of each of the circular holes on the top plate. These springs hold the humidity chambers down in the rack while permitting easy one-handed access to individual chambers. The chambers sit on an open grid shelf above the heaters and magnetic agitator. This shelf is supported by two longitudinal ledges within the bath.

Auxiliary equipment for operation of the constant temperature bath includes a temperature controller (with associated resistance thermometer sensor), heaters, stirrer (centrifugal), chiller (mechanical refrigerator), magnetic agitator, and water feed (solenoid valve). These items are wired to a control cabinet located on the second shelf of the cart. The temperature controller also occuples the second shelf and the chiller is on the bottan shelf. These six auxiliary items are removable or replaceable and individually plug into appropriate outlets on an electrical outlet strip located on the second shelf. Not only are all plugs and outlets labeled, but they are all distinctive, such that no plug will go into an inappropriate outlet.

Other auxiliary equipment include a liquid level controller, a water pressure reducer and a water filter mounted on the end of the bath as shown in figure 1. 
The control cabinet contains a master power switch and associated red signal lamp which controls all power to the apparatus. The control cabinet also contains 6 individual switches and associated signal lights for control of each of the auxillary electrical items as shown in the circuit diagram (Figure 4). The control cabinet also contains three 15-ampere slow blow fuses to protect the equipment.

\section{Operation}

Two connections must be made before the equipment is operational. The green water feed hose must be connected to a water source and the power cord must be connected to a 20-ampere 5-wire 208-volt 3-phase power source. The water feed hose is connected by means of a 1/4-inch hose shank to which the hose is clamped. When the water source is turned on nothing will happen until the main power switch and the water feed switch are turned on. When the water source, main power switch and water feed switch are all on, the bath will fill with water to the proper level and this level will be maintained. The bath drain valve should of course be closed during this operation.

The water feed system has been designed to operate with water pressures up to $2 \mathrm{MPa}$ (300 psi), thereby allowing the use of any conventional water supply. The design is such that temperature perturbations have been minimized by limiting the water flow into the bath during operation. This design results in a very slow fill rate through the automatic system and will require a number of hours to fill the bath through this system. Of course, more rapid filling can be achieved by filling the bath through the top by other means. 
When the water has reached the level of the top plate of the rack, the temperature control equipment can be put into operation. Whenever the temperature control equipment is in operation the stirrer should be on, not only to insure proper temperature control operation but also to prevent overheating of the heaters. It is for this reason that the signal light on the stirrer is green in order to suggest that it be on at all times. The stirrer will not be harmed by operation without fluid in the bath although there is no benefit to be derived from such operation.

The chiller should not be used when the bath liquid temperature exceeds ambient temperature by more than 2 degrees Celsius. Not only is use of the chiller unnecessary under these conditions, but the chiller can be harmed by operation with the evaporator coll at high temperature. There is a separate switch on the chiller itself, which for convenience should be left on at all times. This retains control of the chiller at the control cabinet. Likewise, there are switches on the temperature controller and at the end of the bath for the magnetic agitator which should be left on at all times in order to maintain all control at the control cabinet. 
In all controlled-temperature operation, the temperature controller has to be on. The controller has a ten-position coarse setting, a tenposition medium setting and a continuous ten-turn fine setting scaled in 1000 units. With use, it will be appropriate to determine a calibration curve for the settings. Roughly, there is 1.5 deg $C$ between adjacent medium setting points, $20 \mathrm{deg} \mathrm{C}$ between coarse setting points 4 and 5 and $22 \mathrm{deg} \mathrm{C}$ between coarse setting points 5 and 6 . There is between .005 and .01 deg $C$ per unit of the fine setting. A setting of 4-5-000 gives approximately $5{ }^{\circ} \mathrm{C}$ and 6-6-150 gives approximately $50{ }^{\circ} \mathrm{C}$.

Although the chiller will operate without the temperature controller turned on the heater circuit has been intentionally wired so that it can not be activated without the temperature controller. This will be apparent, since the heater signal light will not be on without the controller signal light being on. The separate heater switch is retained to allow adjustment of the temperature controller without the heaters coming on when this is desired.

When the chiller is in use, it is advisable to have the magnetic agitator in operation as well as the centrifugal stirrer. Minor magnetic agitation suffices to provide the additional mixing desireable and adjustment can be made by means of the pulse duration and pulse frequency controls at the end of the constant temperature bath. Maximum pulse. frequency is beneficial but the pulse duration can be adjusted to give a just audible sound. The magnetic agitator signal light on the control cabinet indicates when power is available to the magnetic agitator system. The indicator light on the end of the constant temperature bath indicates the actual pulsing of the agitator. Similarly, the heater signal light on the control cabinet indicates the avallability 
of power for the heater while the light on the temperature controller indicates the actual application of this power to the heater.

A cover is provided for the constant temperature bath, but is not required for good temperature control. Its main function is to reduce evaporation from and contamination of the bath fluid.

Salt solutions should be added to the humidity chambers to a depth of between one and two $\mathrm{cm}$. These salt solutions should contain considerable excess salt, resulting in a solution with a consistency that approximates a slush. The test samples are held above the salt solution on the two shelves provided in the lid assembly. A convenient method of holding the test samples would be in crystallizing dishes of approximately $55 \mathrm{~mm}$ diameter and $15 \mathrm{~mm}$ depth. The dishes are firmly retained on the shelves by the chain supports while their flexibility allows easy placement or removal of the dishes.

When not in use, the humidity chambers should not be tightly sealed. This will prevent the seal from adhering strongly to the glass. Where not objectionable, a silicone stopcock grease can be used on the gasket to help sealing while reducing adhesion to the glass. The glass 11 p and the gasket should have no extraneous material on them when used. Although every effort has been made to insure leaktightness, any noticeable increase in level of the salt solution should be taken as an indication of leakage. 
When the chambers are sealed for long periods, especially at elevated temperatures, there may be a tendency for the gasket to adhere to the glass. The lid must then be gently raised so as not to upset the test samples. A suction cup with attached handle is provided for this purpose. The cup is attached to the lid and the seal is broken with a tilting action which breaks the seal.

Figure 5 shows some of the saturated salt solutions that are available for use in this type of apparatus along with their approximate relative humidity as a function of temperature. The uncertainty in relative humidity involves three factors, namely:

1) the uncertainty in the equilibrium relative humidity of the salt solutions,

2) the uncertainty in the temperature of the salt solution and

3) the uncertainty in the temperature of the test sample.

Further analysis will be required to establish definitive estimates of uncertainties in the equilibrium relative humidities for the various possible saturated salt solutions. Our present estimates are that the relative humidities shown for the saturated solutions in figure 4 are uncertain to less than $1 \%$. 
The constant temperature bath was operated with 28 humidity chambers in position at 5,25 and $53^{\circ} \mathrm{C}$. Temperature measurements were made in what would have been salt solutions under normal operation at two extreme locations in the bath at ten minute intervals over long periods of time. Analysis of the data, which consisted of over 100 points at each temperature, indicated that the mean difference between the two positions was less than $0.02{ }^{\circ} \mathrm{C}$ for the three temperatures and that three standard deviations of the temperature at one of the locations was less than $0.02{ }^{\circ} \mathrm{C}$ for the three temperatures.

The mean difference and the standard deviation indicate the uncertainty in the known temperature of the salt solution during normal operation. It is appropriate to analyze the effect such an uncertainty will have on the uncertainty of the relative humidity within the chamber. The effect of this uncertainty is related only to the slope of the relative humidity verses temperature curve. The maximum slope of any of the salt solutions shown in figure 5 is associated with $\mathrm{Mg}\left(\mathrm{NO}_{3}\right)_{2}$ at $50^{\circ} \mathrm{C}$ where the slope $1 \mathrm{~s}-.336 \% \mathrm{rh}$ per degree Celsius. Taking the extreme condition of $.04^{\circ} \mathrm{C}$ which is the sum of the mean difference plus three sigma we would have an uncertainty in $\mathrm{rh}$ of $.01 \%$. This is the extreme condition for uncertainty in the salt solution temperature.

The most appropriate location in which to measure the temperature has not been determined. It is 1ikely that a measurement in the bath fluid near the centrifugal stirrer will give a representative temperature for the salt solutions Further measurements should be made to verify this assumption. 
The third uncertainty is related to temperature difference between the salt solution and the test sample. The amount of water vapor in the gas above the salt solution is a function of the salt solution temperature and at least to a first approximation is independent of the temperature of the gas above the solution. The effective relative humidity to which the test sample is exposed is a function of the amount of water vapor in the gas and the temperature of the test sample. Therefore, the relative humidity to which the sample is exposed has a further uncertainty related to the temperature difference between the salt solution and the test sample.

Adequate measurements were not made to establish definitively this uncertainty. Since the entire chamber is immersed in the bath fluid, it seems reasonable to assume that the variability in the temperature of the salt solution represents a greater value than the temperature difference between the salt solution and the test sample. Let us use three sigma (0.2 deg C) as the maximum difference. From this we can establish the maximum uncertainty in effective relative humidity to which the sample is exposed due to this temperature difference. This uncertainty is a function of the absolute temperature and the relative humidity and varies for a .02 deg $C$ temperature difference from 1 part per thousand at $53{ }^{\circ} \mathrm{C}$ to 14 parts in ten thousand at $5{ }^{\circ} \mathrm{C}$. The maximum uncertainty in effective $\mathrm{rh}$, for this temperature difference would therefore be $.14 \%$ for $5{ }^{\circ} \mathrm{C}$ and $100 \%$ rh. 
It would appear that the dominating uncertainty in experiments with this apparatus will be primarily due to the uncertainty in the knowledge of the equilibrium rh for the saturated salt solutions and secondarily due to temperature differences occurring between the salt solutions and the test samples. 


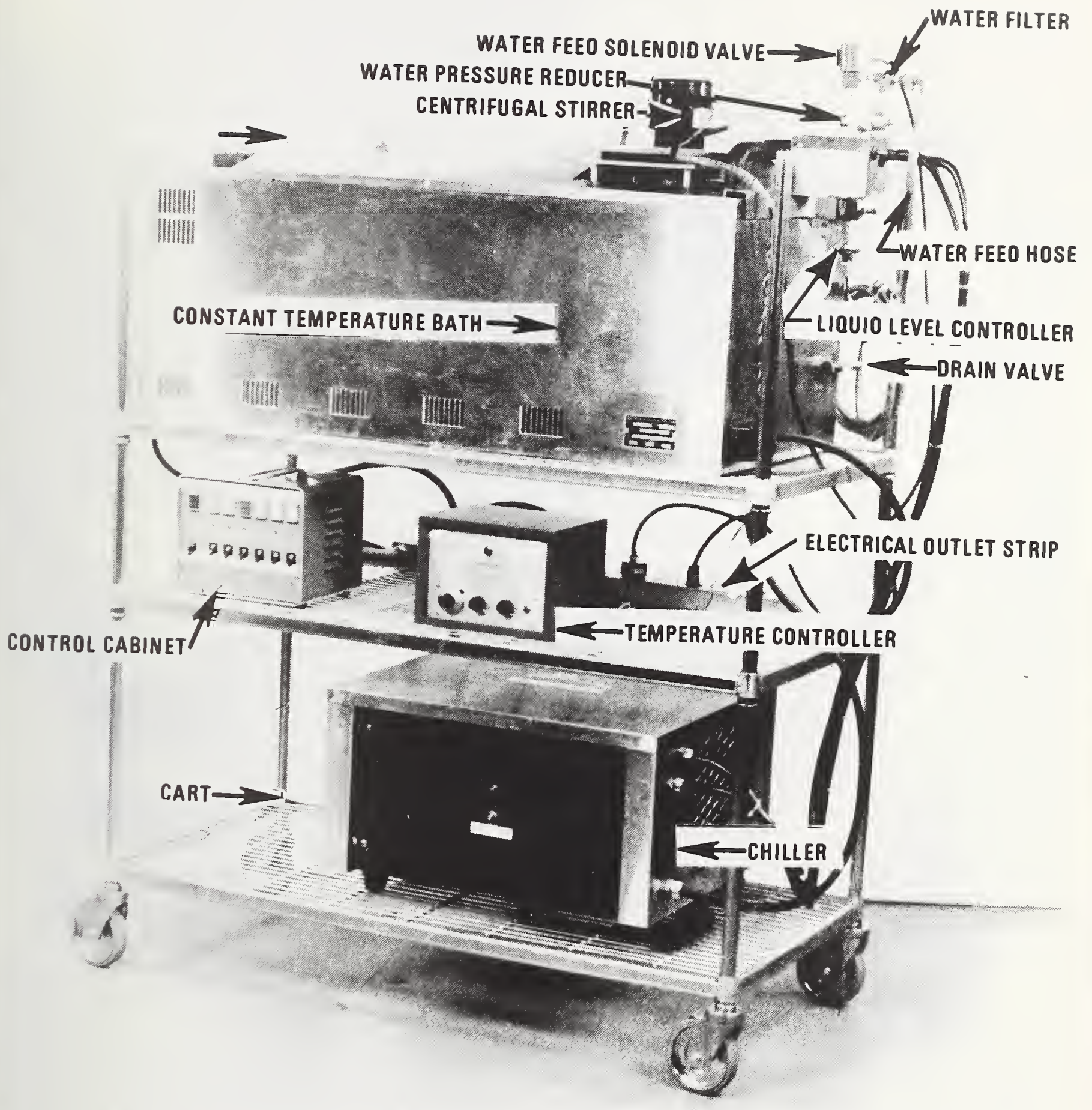

\section{MULTIPLE-CHAMBER HUMIDITY APPARATUS}

Figure 1 


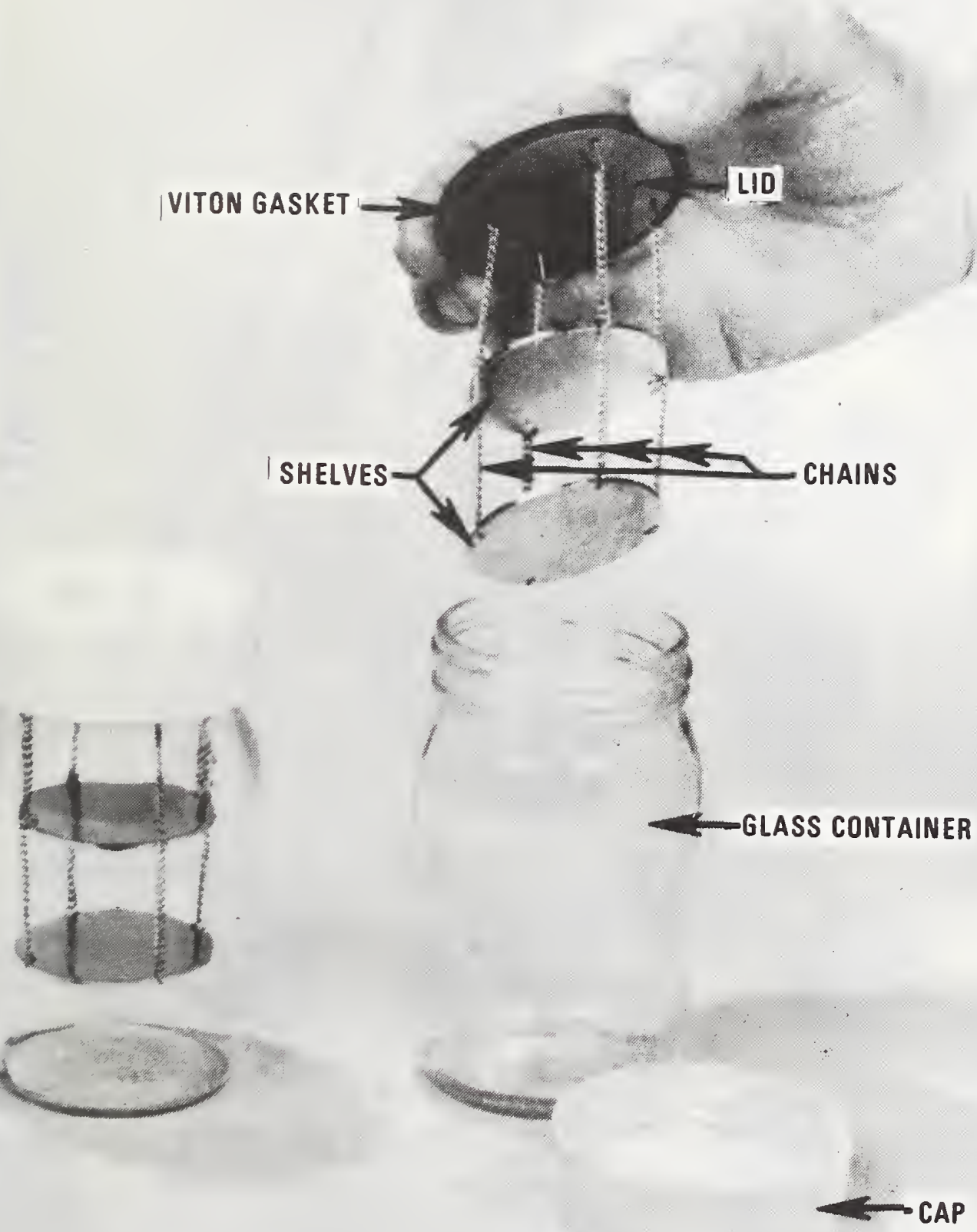

\section{HUMIDITY CHAMBER}

Figure 2 



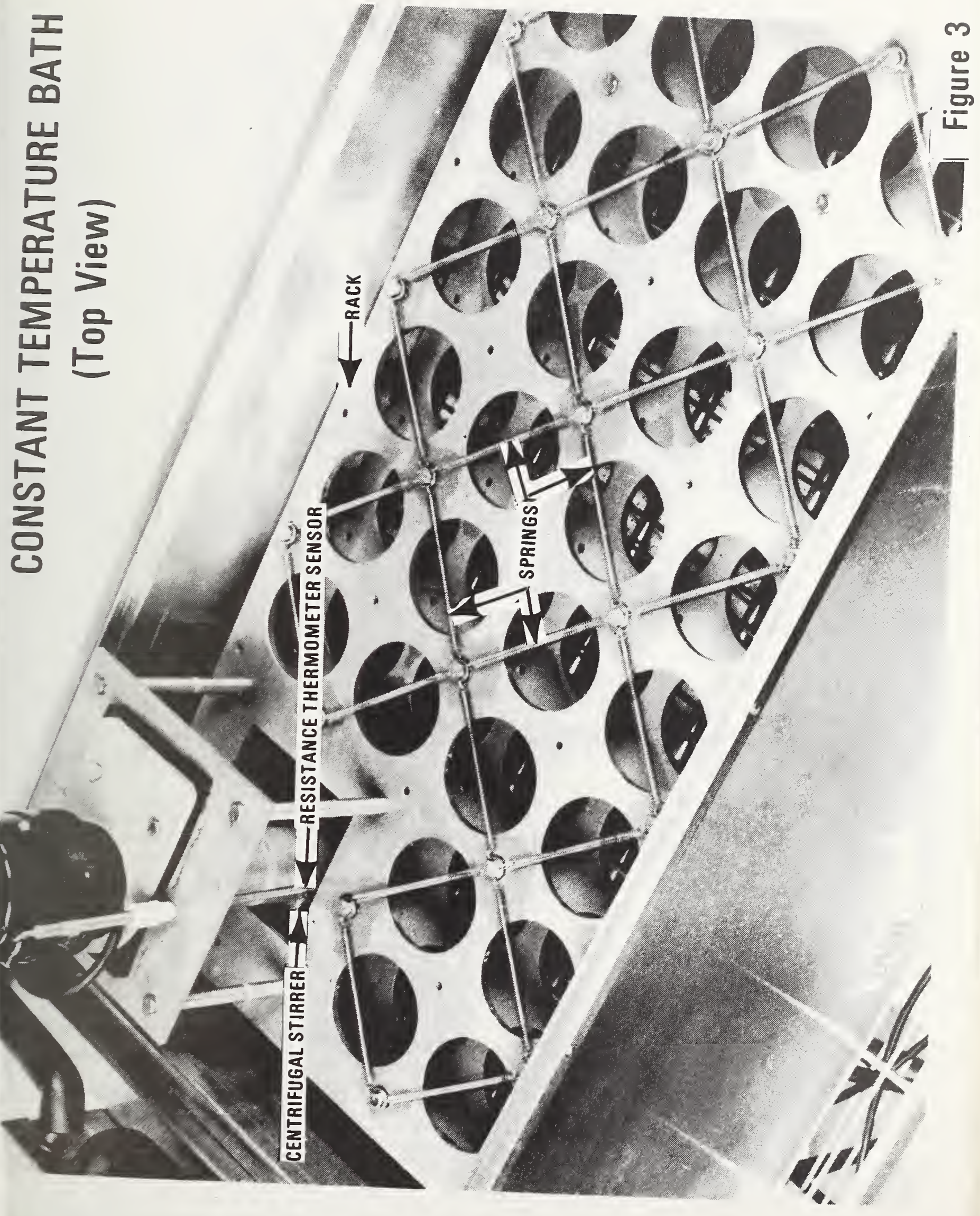





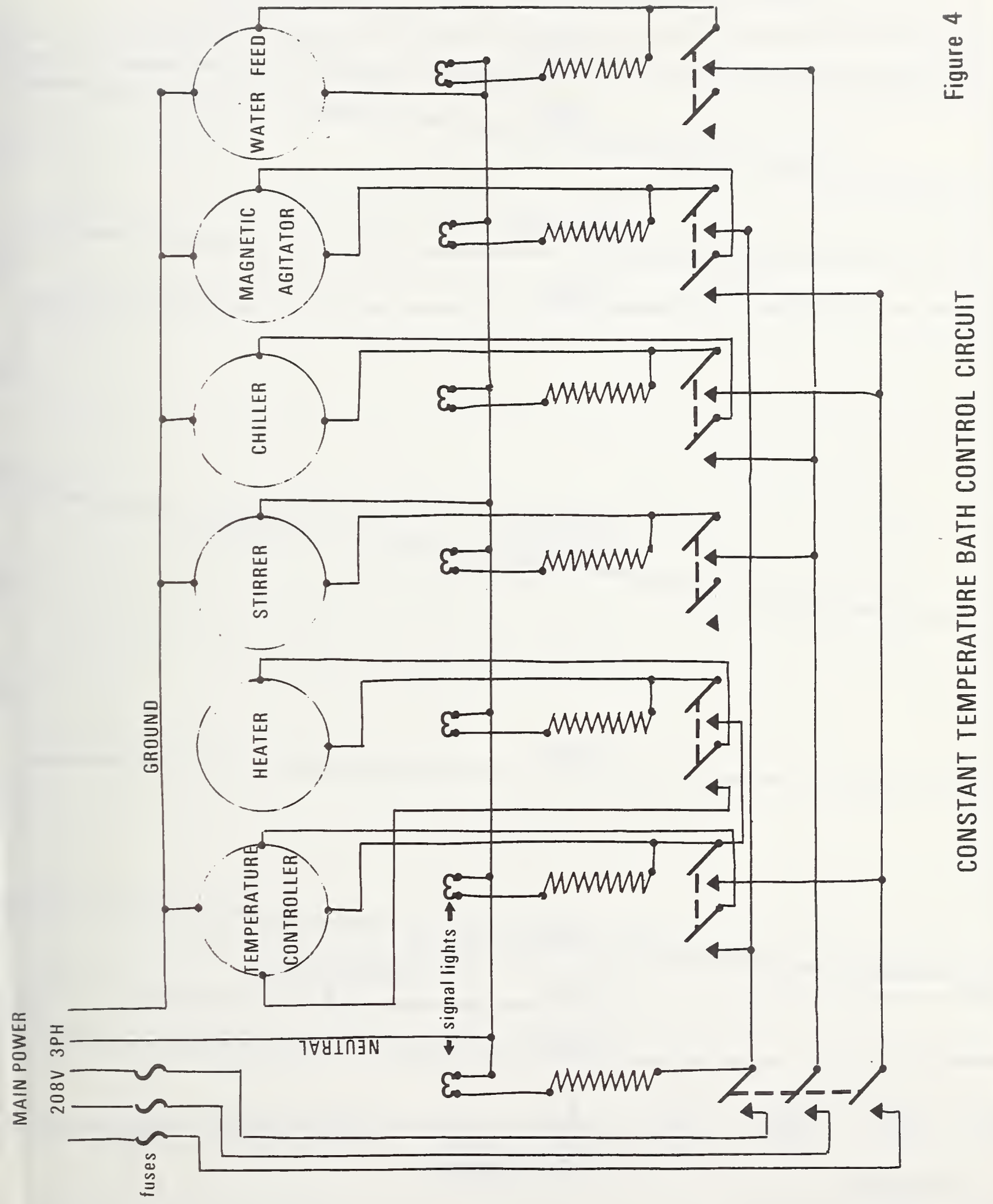



NBS-114A (REV.7.73)

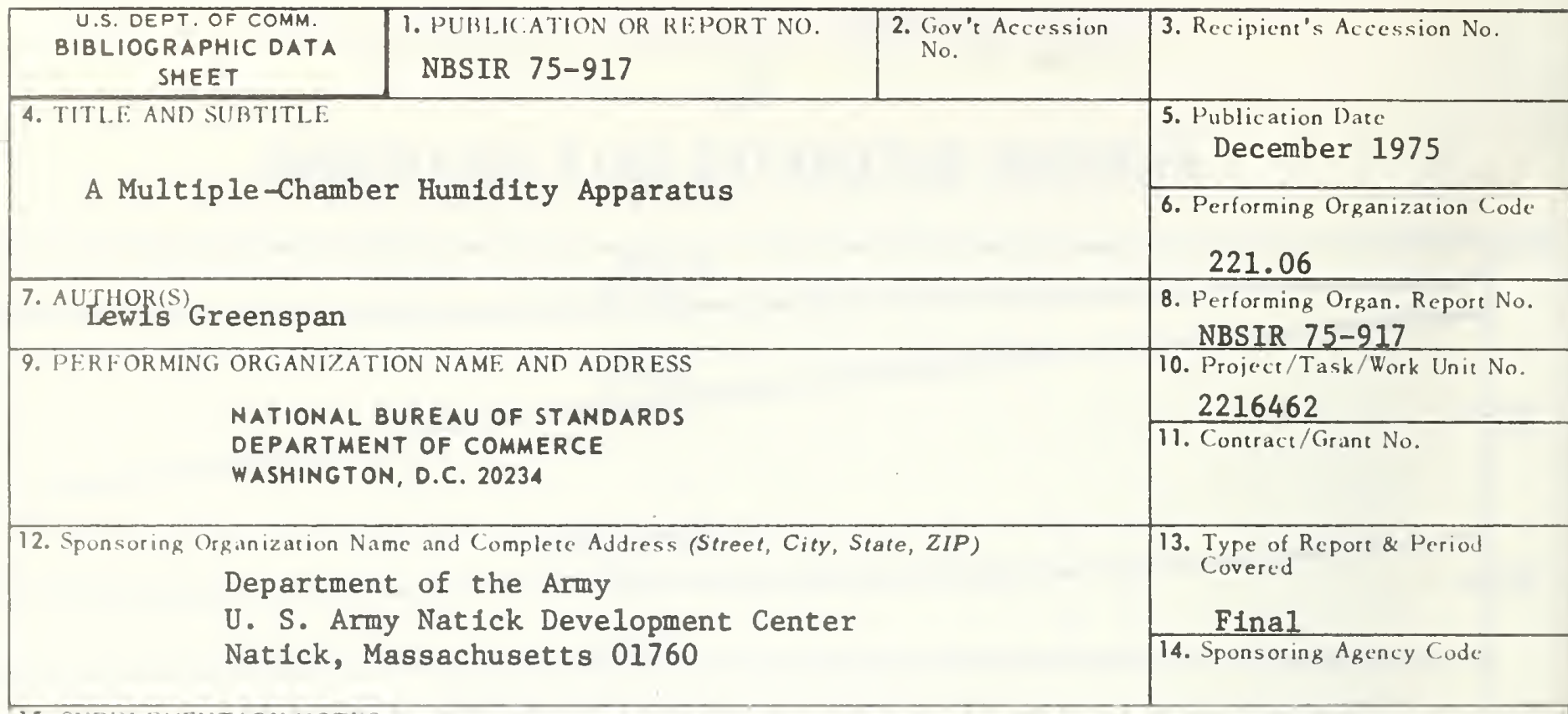

15. SUPPI.EMENTARY NOTES

16. ABSTRACT (A 200-word or less factual summary of most significant information. If document includes a significant bibliography or literature survey, mention it here.)

An apparatus has been developed for studying the growth of microbiological organisms on food under controlled conditions of humidity and temperature. This apparatus contains twenty eight individual humidity chambers within a temperature controlled bath. A wide range of humiditles can be provided within the individual chambers by means of saturated salt solutions. These chambers can be maintained at stable temperatures from 5 to $50{ }^{\circ} \mathrm{C}$ with a constancy and uniformity within $\pm .02 \mathrm{deg} C$. Individual chambers may be conveniently removed or changed without affecting the other chambers.

17. KEY WORDS (six to twelve entries; alphabetical order; capitalize only the first letter of the first key word unless a proper name; separated by semicolons) Humidity; humidity chamber; microbiological growth; relative humidity; salt solutions; saturated salt.

X Unlimited

For Official Distribution. Do Not Release to NTIS

Order From Sup. of Doc., U.S. Government Printing Office

Washington, D.C. 20402, SD Cat. No.C.13

2 Order From National Technical Information Service (NTIS) Springfield, Virginia 22I51

\begin{tabular}{|c|c|}
\hline $\begin{array}{l}\text { 19. SECURITY CLASS } \\
\text { (THIS REPURT) }\end{array}$ & 21. NO. OF PAGES \\
\hline UNCL ASSIFIED & 21 \\
\hline $\begin{array}{l}\text { 20. SECURITY C.LASS } \\
\text { (THIS PAGE) }\end{array}$ & 22. Price \\
\hline UNCLASSIFIED & $\$ 3.25$ \\
\hline
\end{tabular}





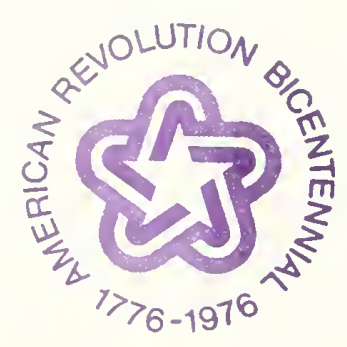

